\title{
PERSISTENTLY FINITE THEORIES WITH HYPERARITHMETIC MODELS
}

\author{
BY
}

TERRENCE MILLAR

\begin{abstract}
Nerode asked if there could be a complete decidable theory with only finitely many countable models up to isomorphism, such that not all of the countable models were decidable. Morley, Lachlan, and Peretyatkin produced examples of such theories. However, all the countable models of those theories were decidable in $0^{\prime}$. The question then arose whether all countable models of such theories had to be, for example, arithmetic. In this paper we provide a negative answer to that question by showing that there are such examples with countable models of arbitrarily high hyperarithmetic degree. It is not difficult to show that any countable model of a hyperarithmetic theory which has only finitely many countable models must be decidable in some hyperarithmetic degree.
\end{abstract}

Preliminaries. For elements $\eta, \xi \in \omega^{<\omega}$ we write $\eta<\xi$ to mean that $\eta(i)=\xi(i)$ for all $i<\operatorname{lh}(\eta)$. We write $\eta<_{L} \xi$ if either $\eta<\xi$ and $\eta \neq \xi$, or $\eta(i)<\xi(i)$ for the least $i$ such that $\eta(i) \neq \xi(i)$. If $B \subset \omega$, then a structure $\mathcal{Q}$ is decidable exactly in $B$ if $\mathscr{Q}$ is decidable relative to $B$ and, whenever $Q$ is decidable relative to $C \subset \omega$, then $B \leqslant{ }_{T} C$. Thus, for example, if $\mathscr{Q}$ is decidable, then $\mathscr{Q}$ is decidable exactly in any recursive $B$. A complete theory $T$ is persistently finite if every complete extension in $L(T) \cup\left\{c_{1}, \ldots, c_{n}\right\}$ has finitely many countable models up to isomorphism, $n<\omega$. The remainder of the notational conventions are more or less standard and can be determined from [1, 2, and 4].

We will now state the result:

THEOREM. For every $n<\omega$ there is a complete decidable persistently finite $T$ such that:

(1) Not every countable model of $T$ is decidable; and

(2) every countable model of $T$ is either decidable exactly in $H(n)$ or is decidable.

Proof. By Kreisel [4] fix a recursive tree $\operatorname{Tr} \subset \omega^{<\omega}$ such that:

(1) Tr has exactly one infinite branch $f^{*} \in \omega^{\omega}$; and

(2) $f^{*} \equiv_{T} H(n)$.

Without loss we will assume that if for some $i<\omega, \eta^{\wedge}\langle i\rangle \in \operatorname{Tr}$, then $\eta^{\wedge}\langle j\rangle \in \operatorname{Tr}$ for all $j<\omega$. We will first specify the language of the desired theory $T$, and then define its prime model. The following will be unary predicate symbols: $\left\{U_{\eta}, S_{\eta}, B_{\eta} \mid \eta \in \operatorname{Tr}\right\}$.

Received by the editors October 21, 1981.

1980 Mathematics Subject Classification. Primary 03C57; Secondary 03C50, 03C65.

${ }^{1}$ The preparation of this paper was partially supported by Grant NSF-MCS-7900824.

(C)1983 American Mathematical Society 0002-9947/82/0000-0795/\$02.75 
Also there will be the following binary predicate symbols: $\left\{E_{\xi}^{\eta}, R_{\xi}^{\eta}, L_{\xi}^{\eta},<\right.$ $\left.\mid \eta^{\wedge}\langle 0\rangle, \xi^{\wedge}\langle 0\rangle \in \operatorname{Tr}\right\}$. The universe of the prime model will be the rationals $Q$. We first define $S_{\eta}^{\mathfrak{Q}}, U_{\eta}^{\mathfrak{Q}}$, and $E_{\eta}^{\mathfrak{Q}}$, by induction on the length of $\eta$.

$$
\operatorname{lh}(\eta)=0: S_{\langle\rangle}^{Q}={ }_{\mathrm{df}} U_{\langle\rangle}^{Q}={ }_{\mathrm{df}} Q .
$$

Fix some onto $f_{\langle\rangle}: Q \rightarrow Q$ such that for all $p, q \in Q$,

(1) $p<q \rightarrow f_{\langle\rangle}(p) \leqslant f_{\langle\rangle}(q)$ and

(2) $f^{-1}(p) \cong Q$.

Then

$$
E_{\langle\rangle}^{Q}=_{\mathrm{df}}\left\{\langle p, q\rangle \mid p, q \in Q, f_{\langle\rangle}(p)=f_{\langle\rangle}(q)\right\} .
$$

$\operatorname{Lh}\left(\eta^{\hat{}}\langle i\rangle\right)=n+1$ : Let $\left\{S_{\eta^{\wedge}\langle i\rangle}^{\mathbb{Q}} \mid i<\omega\right\}$ be defined so that:

(1) $\left\{S_{\eta^{\wedge}\langle i\rangle}^{\mathbb{Q}} \mid i<\omega\right\}$ partitions $U_{\eta}^{\mathbb{Q}}$;

(2) $\forall a, a^{\prime} \in U_{\eta}^{\mathbb{Q}} \forall i<\omega\left[\left\langle a, a^{\prime}\right\rangle \in E_{\eta}^{\mathbb{Q}} \wedge a \in S_{\eta^{\prime}\langle i\rangle}^{\mathbb{Q}} \rightarrow a^{\prime} \in S_{\eta^{\prime}\langle i\rangle}^{\mathbb{Q}}\right]$;

(3) $\forall a, a^{\prime} \in U_{\eta}^{\mathbb{Q}} \forall i<\omega\left[a \in S_{\eta^{\prime}\langle i\rangle}^{\mathbb{Q}} \wedge a^{\prime} \in S_{\eta^{\wedge}\langle i+1\rangle}^{\mathbb{Q}} \rightarrow a<a^{\prime}\right]$; and

(4) $\forall i<\omega\left[f_{\eta}^{\prime \prime}\left[S_{\eta^{\prime}\langle i\rangle}^{Q}\right] \cong Q\right]$.

By (4) fix a $g_{n^{\wedge}\langle i\rangle}: Q \stackrel{\equiv}{\Rightarrow} f_{\eta}^{\prime \prime}\left[S_{\eta^{\wedge}\langle i\rangle}^{Q}\right]$ for each $i<\omega$. Now define $U_{\eta^{\wedge}\langle i\rangle}^{Q}={ }_{\mathrm{df}} f_{\eta}^{-1}\left(g_{\eta^{\wedge}\langle i\rangle}(0)\right)$ for all $i\left\langle\omega\right.$. If $\eta^{\wedge}\langle i\rangle\langle 0\rangle \notin \operatorname{Tr}$, then we are done. Otherwise, for each such $i$ fix an $f_{\eta^{\wedge}\langle i\rangle}: U_{\eta^{\wedge}\langle i\rangle}^{\mathbb{Q}} \rightarrow Q$ such that:

(1) $\forall p, q \in U_{\eta^{\wedge}\langle i\rangle}^{Q}\left[p<q \rightarrow f_{\eta^{\wedge}\langle i\rangle}(p) \leqslant f_{\eta^{\wedge}\langle i\rangle}(q)\right]$; and

(2) $\forall p \in Q\left[f_{\eta \dot{\gamma}\langle i\rangle}^{-1}(p) \cong Q\right]$.

Then

$$
E_{\eta^{\hat{\prime}\langle i\rangle}}^{\mathbb{Q}}={ }_{\mathrm{df}}\left\{\langle p, q\rangle \mid p, q \in U_{\eta^{\hat{\eta}\langle i\rangle}}^{\mathbb{Q}}, f_{\hat{\eta}^{\hat{\eta}\langle i\rangle}}(p)=f_{\eta^{\hat{\imath}\langle i\rangle}}(q)\right\} .
$$

Next, the other predicate symbols' interpretations:

$B_{\langle\rangle}^{\mathfrak{Q}}={ }_{\mathrm{df}} \varnothing$, and for $\eta \neq\langle\rangle, B_{\eta}^{\mathbb{Q}}={ }_{\mathrm{df}}\left\{p \in S_{\eta}^{\mathfrak{Q}} \mid g_{\eta}^{-1}\left(f_{\eta \mathfrak{l}} \ln \eta-1(p)\right)<0\right\}$;

$\left(R_{\xi}^{\eta}\right)^{\mathbb{Q}}={ }_{\mathrm{df}}\left\{\langle p, q\rangle \mid p \in S_{\eta}^{\mathbb{Q}}, q \in S_{\xi}^{\mathbb{Q}}\right.$, and $\left.f_{\eta}(p)=f_{\xi}(q)\right\}$;

$\left(L_{\xi}^{\eta}\right)^{\mathbb{Q}}={ }_{\mathrm{df}}\left\{\langle p, q\rangle \mid p \in S_{\eta}^{\mathbb{Q}}, q \in S_{\xi}^{\mathbb{Q}}\right.$, and $\left.f_{\eta}(p)<f_{\xi}(q)\right\}$; and

$<^{Q}={ }_{\mathrm{df}}<Q$.

The reduct $\beta$ of $\mathcal{Q}$ to the signature $\left\{<, S_{\eta} \mid \eta \in \operatorname{Tr}\right\}$ is thus a dense linear order with $\operatorname{Tr}$ coded in via the $S_{\eta}$ 's and ordered lexicographically. Also note that the theory of $\beta$ has a type $\Gamma$ that satisfies $f^{*} \leqslant_{T} \Gamma$, i.e. any type $\Gamma$ containing $\left\{S_{f^{*} \mid n}(x) \mid n<\omega\right\}$. The problem with $\beta$ is that its theory has continuumly many countable models. The way to remedy this fault is either to eliminate the other, "nonessential" nonprincipal types, or to tie their realizations together. The first option is not possible, since it is straightforward to show that a bound on the Cantor-Bendixson rank of the types of a theory places a corresponding bound on the Turing complexity of those types. Thus, for instance, if a theory has a nonarithmetic type, then it must have types of all finite Cantor-Bendixson ranks. So, unless the realizations of such types are linked, there are necessarily continuumly many countable models of the underlying theory.

On the other hand, the method chosen to connect the realizations of different types is subject to several constraints. First, it is easy to show that if whenever a type $\Gamma$ is realized in a model, a type $\Sigma$ must also be realized, then $\Sigma \leqslant_{T} \Gamma$. Also, if the 
Cantor-Bendixson rank of $\Gamma$ is less than that of $\Sigma$, then the method used to connect realizations cannot be as simple as a function. This is the explanation of the $R_{\xi}^{\eta}$ 's. In

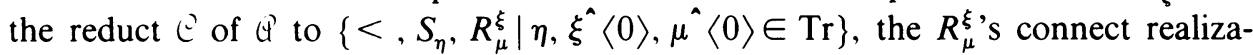
tions of the two types containing $\left\{S_{\xi}(x), \neg S_{\xi\langle i\rangle}(x) \mid i<\omega\right\},\left\{S_{\mu}(x), \neg S_{\mu \wedge i\rangle}(x) \mid i<\right.$ $\omega)$ respectively. The $E_{\eta}$ 's are included to obtain a theory that eliminates quantifiers, since the elements $R_{\xi}^{\eta}$-related to a given element are thus certainly related to each other. Similarly the $L_{\xi}^{\eta}$ 's are needed for the elimination of quantifiers, since they reflect the $<$ order within $S_{\eta}$ "slices". Finally, the $U_{\eta}$ 's pick out the $E_{\eta}$ equivalence set which is used to code the extensions of $\eta$ in $\operatorname{Tr}$, and the $B_{\eta}$ 's are added for the elimination of quantifiers to denote being < -before the corresponding $U_{\eta}$ 's. We now proceed to show that the theory of $\mathbb{Q}$ allows an elimination of quantifiers.

Let the following set of universal axioms constitute a theory $T^{\prime}$ : For all $\eta, \xi$ and $\mu$ for which the corresponding predicate symbols are in $L(T)$ :

(I) " $<$ is a linear ordering of the universe";

(II) $S_{\langle\rangle}(x) \wedge \neg B_{\langle\rangle}(x)$;

(III) $S_{\eta}(x) \rightarrow S_{\xi}(x)$ for $\xi<\eta$;

(IV) $S_{\eta}(x) \rightarrow \neg S_{\xi}(x)$ for $\eta<_{\llcorner} \xi$ and $\eta \Varangle \xi$;

(V) $U_{\eta}(x) \rightarrow S_{\eta}(x)$

(VI) $S_{n \hat{\imath}\langle i\rangle}(x) \rightarrow U_{\eta}(x)$;

(VII) " $E_{\eta}$ is an equivalence relation on $U_{\eta}$ ";

(VIII) $S_{\eta^{\hat{\imath}}\langle i\rangle}(x) \wedge S_{\eta^{\wedge}\langle j\rangle}(y) \rightarrow x<y$ for $i<j$;

(IX) $B_{\eta}(x) \rightarrow S_{\eta}(x) \wedge \neg U_{\eta}(x)$;

(X) $B_{\eta}(x) \wedge S_{\eta}(y) \wedge \neg B_{\eta}(y) \rightarrow x<y$;

(XI) $U_{\eta^{\hat{\gamma}\langle i\rangle}}(x) \rightarrow\left[U_{\eta^{\hat{\langle}\langle i\rangle}}(y) \leftrightarrow E_{\eta}(x, y)\right]$;

(XII) $R_{\eta}^{\eta}(x, y) \leftrightarrow E_{\eta}(x, y)$;

(XIII) $R_{\xi}^{\eta}(x, y) \rightarrow U_{\eta}(x) \wedge U_{\xi}(y) \wedge R_{\eta}^{\xi}(y, x)$;

(XIV) $R_{\xi}^{\eta}(x, y) \wedge R_{\mu}^{\xi}(y, z) \rightarrow R_{\mu}^{\eta}(x, z)$;

$(\mathrm{XV}) L_{\xi}^{\eta}(x, y) \rightarrow U_{\eta}(x) \wedge U_{\xi}(y) \wedge \neg R_{\xi}^{\eta}(x, y) \wedge \neg L_{\eta}^{\xi}(y, x)$;

$(\mathrm{XVI}) L_{\xi}^{\eta}(x, y) \wedge L_{\mu}^{\xi}(y, z) \rightarrow L_{\mu}^{\eta}(x, z)$;

(XVII) $S_{\eta}(x) \wedge S_{\eta}(y) \wedge \neg E_{\eta}(x, y) \rightarrow\left[L_{\eta}^{\eta}(x, y) \leftrightarrow x<y\right]$;

(XVIII) $S_{\eta}(x) \wedge S_{\xi}(y) \rightarrow\left[L_{\xi}^{\eta}(x, y) \vee L_{\eta}^{\xi}(y, x) \vee R_{\xi}^{\eta}(x, y)\right]$;

(XIX) $E_{\eta}(x, y) \wedge x<z \wedge z<y \rightarrow E_{\eta}(x, z)$;

$\left(\mathrm{XX)} R_{\xi}^{\eta}(x, y) \wedge U_{\eta^{\wedge}\langle i\rangle}(x) \rightarrow U_{\xi\langle i\rangle}(y)\right.$;

(XXI) $R_{\xi}^{\eta}(x, y) \wedge B_{\eta \hat{\eta}\langle i\rangle}(x) \rightarrow B_{\xi\langle i\rangle}(y)$;

(XXII) $B_{\eta^{\hat{\gamma}\langle i\rangle}}(x) \wedge \neg B_{\xi\langle i\rangle}(y) \wedge S_{\xi\langle i\rangle}(y) \rightarrow L_{\xi}^{\eta}(x, y)$;

(XXIII) $U_{\eta^{\wedge}\langle i\rangle}(x) \wedge S_{\xi\langle i\rangle}(y) \wedge \neg B_{\xi\langle i\rangle}(y) \wedge \neg U_{\xi\langle i\rangle}(y) \rightarrow L_{\xi}^{\eta}(x, y)$;

$\left(\mathrm{XXIV)} R_{\xi}^{\eta}(x, y) \wedge R_{\xi}^{\eta}(u, w) \wedge \neg E_{\eta}(x, u) \rightarrow[x<u \leftrightarrow y<w]\right.$;

$(\mathrm{XXV}) R_{\xi}^{\eta}(x, y) \wedge S_{\eta^{\hat{\langle}}\langle i\rangle}(x) \rightarrow S_{\eta \hat{\eta}\langle i\rangle}(y)$;

$(\mathrm{XXVI}) L_{\xi}^{\eta}(x, y) \wedge S_{\eta^{\wedge}\langle i\rangle}(x) \rightarrow \neg S_{\xi^{\wedge}\langle j\rangle}(y)$ for $j<i$;

$(\mathrm{XXVII}) R_{\xi}^{\eta}(x, y) \wedge U_{\eta}(u) \wedge u<x \wedge \neg E_{\eta}(u, x) \rightarrow L_{\xi}^{\eta}(u, y)$

(XXVIII) $L_{\eta}^{\xi}(y, x) \wedge U_{\eta}(u) \wedge x<u \rightarrow L_{\eta}^{\xi}(y, u)$

$\left(\mathrm{XXIX)} L_{\eta}^{\xi}(y, x) \wedge U_{\xi}(u) \wedge u<y \rightarrow L_{\eta}^{\xi}(u, x)\right.$;

$\left(\mathrm{XXX)} L_{\xi}^{\eta}(x, y) \wedge R_{\mu}^{\xi}(y, z) \rightarrow L_{\mu}^{\eta}(x, z)\right.$;

$(\mathrm{XXXI}) L_{\xi}^{\eta}(x, y) \wedge R_{\eta}^{\mu}(z, x) \rightarrow L_{\xi}^{\mu}(z, y)$. 
It is straightforward to check that $Q \vDash T^{\prime}$-we leave the verification to the reader.

Our next goal is to show that $T$ allows elimination of quantifiers and is decidable. In fact, $T$ is also the model completion of $T^{\prime}$. Let us say that a set of formulas $\Gamma\left(x_{1}, \ldots, x_{n}\right)$ is a basic n-type of a theory if it is a maximal set of quantifier free formulas in the free variables $\left\{x_{1}, \ldots, x_{n}\right\}$ consistent with that theory. By the signature of $L(T)$ :

Lemma 1. Every basic n-type of $T$ is uniquely determined by its 2-type projections.

Next,

LEMMA 2. Each of the following sets of formulas uniquely determines a basic 1-type of $T$; moreover, each basic 1-type of $T$ contains one of these sets:

(1) For each $\eta \in \mathrm{Tr}$ :

(a) $\left\{B_{\eta}(x)\right\}$;

(b) $\left\{\neg B_{\eta}(x), \neg U_{\eta}(x), S_{\eta}(x)\right\}$;

(2) for each $\eta \in \operatorname{Tr}$ such that $\eta^{\wedge}\langle 0\rangle \notin \operatorname{Tr},\left\{U_{\eta}(x)\right\}$;

(3) for each $\eta \in \operatorname{Tr}$ such that $\eta^{\hat{}}\langle 0\rangle \in \operatorname{Tr},\left\{U_{\eta}(x), \neg S_{\eta^{\hat{\eta}}\langle i\rangle}(x) \mid i<\omega\right\}$; and

(4) for $f^{*}$, the infinite branch of $\operatorname{Tr},\left\{S_{f^{*} i}(x) \mid i<\omega\right\}$.

Proof. It is easy to see that each of the above sets of formulas is consistent with $T$. We will show first that each determines a unique basic 1-type. So assume that $\Gamma(x)$ is a basic 1-type containing one of the above. By the axioms in (II)-(IX) there is a unique $h \in \omega^{<\omega} \cup \omega^{\omega}$ of maximum length such that

$S_{h i}(x) \in \Gamma(x)$ for all $i \leqslant \operatorname{lh}(h)$ if $h \in \omega^{<\omega}$, and for all $i<\omega$ otherwise.

Now, again by the axioms in (II)-(IX), $\Gamma$ 's containment of any one of the above sets uniquely determines that $h$. So, by those axioms $\Gamma$ 's containment of all the $U_{\mu}(x), S_{\mu}(x)$ and $B_{\mu}(x)$ is uniquely determined. They also determine exactly which $E_{\mu}(x, x)$ belongs to $\Gamma$. Thus, by the axioms in (XII) and (XIII) the $R_{\mu}^{\sigma}(x, x)$ are uniquely determined. Finally by the axioms in (I), (XV), and (XVII), $\neg(x<x)$ and $\neg L_{\mu}^{\sigma}(x, x)$ belong to all basic l-types of $T$. This proves the first half. Let us denote each of the basic 1-types thus determined by:

(1) (a) $\Gamma_{h}^{B}$;

(b) $\Gamma_{h}^{A}$;

(2) $\Gamma_{h}^{U}$;

(3) $\Gamma_{h}^{L}$; and

(4) $\Gamma_{f^{*}}$, respectively.

Now let $\Gamma(x)$ be any basic l-type of $T$. Let $h \in \omega^{<\omega} \cup \omega^{\omega}$ be the unique element of maximum length such that

$S_{h i}(x) \in \Gamma(x)$ for all $i \leqslant \ln (h)$ if $\ln (h)<\omega$, and $i<\omega$ otherwise. By the choice of $\operatorname{Tr}$, if $h \in \omega^{\omega}$, then $h=f^{*}$, and so in that case $\Gamma=\Gamma_{f^{*}}$. So assume that $h \in \omega^{<\omega}$. Now if $B_{h}(x)$ or $\left[\neg B_{h}(x) \wedge \neg U_{h}(x)\right]$ is in $\Gamma(x)$, then $\Gamma=\Gamma_{h}^{B}, \Gamma=\Gamma_{h}^{A}$, respectively. If not, then $U_{h}(x)$ is in $\Gamma(x)$, and thus by the choice of $h$ either $h^{\wedge}\langle 0\rangle \notin \operatorname{Tr}$ or $\neg S_{h^{\wedge}\langle i\rangle}(x) \in \Gamma(x)$ for all $i<\omega$. In the first case $\Gamma=\Gamma_{h}^{U}$, while for the second $\Gamma=\Gamma_{h}^{L}$. 
Lemma 3. Every basic 2-type of $T$ is uniquely determined by its 1-type projections and finitely many formulas that it contains.

Proof. Let $\Sigma(x, y)$ be a basic 2-type of $T$ and $\Gamma_{g}^{X}(x), \Gamma_{h}^{Y}(y)$ its 1-type projections. By the axioms in (II)-(IX) and (XIII), if $R_{\xi}^{\eta}(x, y) \in \Sigma(x, y)$ then $\eta<g$ and $\xi<h$. Also, if $\eta<g$ and $\xi<_{+}$, then $R_{\xi}^{\eta}(x, y) \in \Sigma(x, y)$ if and only if $g(\operatorname{lh}(\eta))=$ $h(\mathrm{lh}(\xi))$, by the axioms in (II)-(IX), (XX), and (XXV). Similarly, by the axioms in (XV), if $L_{\xi}^{\eta}(x, y) \in \Sigma(x, y)$ then again $\eta<g$ and $\xi<h$. This time, however, if $\mathrm{lh}(\eta)<g$ then by the axioms in (VIII), (XXX), (XV), (XVII), (XX), and (XXXI), $L_{\xi}^{\eta}(x, y) \in \Sigma(x, y)$ if and only if $g(\operatorname{lh}(\eta))<h(\operatorname{lh}(\xi))$. By the axioms in (XII), the $E_{\eta}(x, y)$ 's are also determined. Thus there exists at most eight atomic, negated atomic formulas not uniquely determined by the 1-type projections: $(x=y)^{k}$, $(x<y)^{k}, R_{h}^{g}(x, y)^{k}$, and $L_{h}^{g}(x, y)^{k}, k=0,1$ (of course if $g$ or $h$ is $f^{*}$, or $g^{\wedge}\langle 0\rangle$ or $h^{\wedge}\langle 0\rangle \notin \operatorname{Tr}$, then the corresponding formula does not exist).

Let $L_{N}$ be the restriction of $L(T)$ to those predicate symbols whose subscripts and superscripts $\eta$ satisfying $\sum_{i<\operatorname{lh}(\eta)} \eta(i)+\operatorname{lh}(\eta) \leqslant N$. Similarly, if $\Sigma$ is a set of formulas of $T$, then let $\Sigma \uparrow_{N}$ denote $L_{N} \cap \Sigma$.

Lemma 4. Assume that $\Gamma, \Sigma$ are basic $m$ and $m+1$-types of $T^{\prime} \uparrow_{N}$, respectively, and $\Gamma\left(x_{1}, \ldots, x_{m}\right) \subset \Sigma\left(x_{1}, \ldots, x_{m+1}\right)$. Then

$$
T \vdash \forall x_{1} \forall x_{2} \cdots \forall x_{m}\left[\wedge \Gamma\left(x_{1}, \ldots, x_{m}\right) \rightarrow \exists x_{m+1} \wedge \Sigma\left(x_{1}, \ldots, x_{m+1}\right)\right] .
$$

Proof. Asssume that $\mathbb{Q} \vDash \wedge \Gamma\left(a_{1}, \ldots, a_{m}\right)$ for some $a_{1}, \ldots, a_{m} \in|\mathbb{Q}|$. It then suffices to show that there is an $a \in|Q|$ such that $Q \vDash \wedge \Sigma\left(a_{1}, \ldots, a_{m}, a\right)$. If $\left(x_{i}=x_{m+1}\right) \in \Sigma$ for some $i \leqslant m$, then this is trivial. So assume otherwise. For each $i, 1 \leqslant i \leqslant m+1$, fix the $\eta_{i} \in \operatorname{Tr}$ of greatest length such that $U_{\eta_{i}}\left(x_{i}\right) \in \Sigma$ and $\hat{\eta_{i}}\langle 0\rangle \in \operatorname{Tr}$. Also fix the $\xi$ of greatest length such that $S_{\xi}\left(x_{m+1}\right) \in \Sigma$. Now choose any $a \in|Q|$ such that:

(1) $\mathbb{Q} \vDash S_{\xi}(a)$

(2) $U_{\xi}\left(x_{m+1}\right) \in \Sigma$ if and only if $Q \vDash U_{\xi}(a)$;

(3) $B_{\xi}\left(x_{m+1}\right) \in \Sigma$ if and only if $Q \vDash B_{\xi}(a)$;

(4) $R_{\eta_{m+1}}^{\eta_{i}}\left(x_{i}, x_{m+1}\right) \in \Sigma$ if and only if $Q \vDash R_{\eta_{m+1}}^{\eta_{i}}\left(a_{i}, a\right)$; and

(5) $L_{\eta_{m+1}}^{\eta_{i}}\left(x_{i}, x_{m+1}\right) \in \Sigma$ if and only if $Q \vDash L_{\eta_{m+1}}^{\eta_{i}}\left(a_{i}, a\right)$.

By Lemma 3 and its proof, for any such $a \in|Q|$,

$$
Q \vDash \wedge \Sigma\left(a_{1}, \ldots, a_{m}, a\right) \text {. }
$$

The existence of such an $a \in|Q|$ is easy to verify by the definition of $Q$ and the consistency of $\Sigma$ with $T^{\prime} \uparrow_{N}$. For example, suppose that

$$
L_{\eta_{m+1}}^{\eta_{i}}\left(x_{i}, x_{m+1}\right), L_{\eta_{j}}^{\eta_{m+1}}\left(x_{m+1}, x_{j}\right) \in \Sigma\left(x_{1}, \ldots, x_{m+1}\right) .
$$

Fix $a_{i}^{*}, a_{j}^{*} \in|\mathbb{Q}|$ such that

$$
\mathbb{Q} \vDash R_{\eta_{m+1}}^{\eta_{i}}\left(a_{i}, a_{i}^{*}\right) \wedge R_{\eta_{m+1}}^{\eta_{j}}\left(a_{j}, a_{j}^{*}\right) .
$$

Now, by the consistency of $\Sigma$ with $T^{\prime} \uparrow_{N}$ it follows that, by the axioms in (XVI), $L_{\eta_{j}}^{\eta_{i}}\left(x_{i}, x_{j}\right) \in \Sigma\left(x_{1}, \ldots, x_{m+1}\right)$ and so

$$
\mathbb{Q} \vDash L_{\eta_{j}}^{\eta_{i}}\left(a_{i}, a_{j}\right) \text {. }
$$


Next, by the definition of $Q$ and the fact that it thus satisfies the axioms in (XXX) and $(\mathrm{XXXI})$,

$$
Q \vDash L_{\eta_{m+1}}^{\eta_{m+1}}\left(a_{i}^{*}, a_{j}^{*}\right)
$$

Thus by the property expressed in (XVII) either

$$
Q \vDash E_{\eta_{m+1}}\left(a_{i}^{*}, a_{j}^{*}\right) \text { or } Q \vDash a_{i}^{*}<a_{j}^{*} .
$$

Now the first is impossible because otherwise two applications of (XIV) would give $Q \vDash R_{\eta_{j}}^{\eta_{i}}\left(a_{i}, a_{j}\right)$, which would violate the property expressed in $(\mathrm{XV})$. Finally, by the definition of $\mathcal{Q}$, there is an $a \in|\mathbb{Q}|$ satisfying

$$
\mathbb{Q} \vDash a_{i}^{*}<a \wedge a<a_{j}^{*} \wedge \neg E_{\eta_{m+1}}\left(a, a_{i}^{*}\right) \wedge \neg E_{\eta_{m+1}}\left(a, a_{i}^{*}\right) .
$$

It is then easy to see that for such an $a \in|\mathscr{Q}|$, by properties (XIX) and (XVIII),

$$
Q \vDash L_{\eta_{m+1}}^{\eta_{i}}\left(a_{i}, a\right) \wedge L_{\eta_{j}}^{\eta_{m+1}}\left(a, a_{j}\right) .
$$

We leave the rest of the details of the proof of this lemma to the reader.

LEMMA 5. $T$ allows an effective elimination of quantifiers.

Proof. It is enough to show that uniformly effectively in quantifier free $\varphi\left(x_{1}, \ldots, x_{m}, y\right)$ there exists a quantifier free $\theta\left(x_{1}, \ldots, x_{m}\right)$ such that

$$
T \vdash \exists y \varphi\left(x_{1}, \ldots, x_{m}, y\right) \leftrightarrow \theta\left(x_{1}, \ldots, x_{m}\right) .
$$

Fix such a $\varphi\left(x_{1}, \ldots, x_{m}\right)$ and let $N$ be the smallest integer such that $\varphi\left(x_{1}, \ldots, x_{m}, y\right)$ is in $L(T) \uparrow_{N}$. First check the consistency of $T^{\prime} \uparrow_{N} \cup\left\{\varphi\left(x_{1}, \ldots, x_{m}, y\right)\right\}$. If this is inconsistence, then $\theta={ }_{\mathrm{df}} \perp$ (a 0 -ary predicate symbol introduced for "false"). Otherwise let $\Sigma_{i}\left(x_{1}, \ldots, x_{m}, y\right), i<M$, be all basic $m+1$-types of $T^{\prime} \uparrow_{N}$ such that $\varphi\left(x_{1}, \ldots, x_{m}, y\right) \in \Sigma_{i}\left(x_{1}, \ldots, x_{m}, y\right)$. For each $i<M$ let $\Gamma_{i}\left(x_{1}, \ldots, x_{m}\right) \subset$ $\Sigma_{i}\left(x_{1}, \ldots, x_{m}, y\right)$ be the corresponding basic $m$-type of $T^{\prime} \uparrow_{N}$. Now

$$
T^{\prime} \uparrow_{N} \vdash \varphi\left(x_{1}, \ldots, x_{m}, y\right) \leftrightarrow \underset{i<M}{\vee} \wedge \Sigma_{i}\left(x_{1}, \ldots, x_{m}, y\right) .
$$

So the two formulas are equivalent under $T$ also. By Lemma 4,

$$
T \vdash \exists y \wedge \Sigma_{i}\left(x_{1}, \ldots, x_{m}, y\right) \leftrightarrow \Gamma_{i}\left(x_{1}, \ldots, x_{m}\right) \text { for } i<M .
$$

Therefore

$$
T \vdash \exists y \varphi\left(x_{1}, \ldots, x_{m}, y\right) \leftrightarrow \underset{i<M}{\bigvee} \wedge \Gamma_{i}\left(x_{1}, \ldots, x_{m}\right),
$$

and so we take $\theta\left(x_{1}, \ldots, x_{m}\right)$ to be $\bigvee_{i<M} \wedge \Gamma_{i}\left(x_{1}, \ldots, x_{m}\right)$.

By Lemmas 4 and 5, $T$ is decidable and allows elimination of quantifiers. In fact, by the above lemmas it is easy to see that $T$ is the model completion of $T^{\prime}$. We will now show that $T$ has only finitely many countable models up to isomorphism. First note that because $T$ allows elimination of quantifiers, each basic $n$-type of $T$ determines a unique $n$-type of $T$ through containment. Therefore let $\Gamma_{g}^{X}$ denote the 1-type of $T$ that contains the basic 1-type $\Gamma_{g}^{X}$ of Lemma 2. Now, in every model $\mathbb{Q}$ of $T$, exactly one of the following holds:

(I) (a) $\mathbb{Q}$ omits $\Gamma_{\zeta\rangle}^{L}$; 
(b) there is an $a \in\left|\vec{t}^{\prime}\right|$ realizing $\Gamma_{\langle}^{L^{L}}$, such that if $a^{\prime} \in|\mathbb{Q}|$ realizes $\Gamma_{\langle>}^{\left.{ }^{L}\right\rangle}$, then $u^{\prime} \vDash\left[E_{\langle\rangle}\left(a, a^{\prime}\right) \vee a<a^{\prime}\right]$;

(c) otherwise.

Also, exactly one of the following holds:

(II) (a) $Q^{*}$ omits $\Gamma_{f^{*}}$;

(b) (ै' realizes $\Gamma_{f^{*}}$ but omits $\Gamma_{f^{*}}(x) \cup \Gamma_{f^{*}}(y) \cup\{x \neq y\}$;

(c) not (i) or (ii) and there is a $<$-least and $<$-greatest realization of $\Gamma_{f^{*}}$;

(d) not (i), (ii), or (iii) and there is a $<$-least realization of $\Gamma_{f^{*}}$;

(e) not (i), (ii), or (iii) and there is a $<$-greatest realization of $\Gamma_{f^{*}}$;

(f) otherwise.

In fact,

LEMMA 6. If two countable models of $T$ satisfy the same options from (I) and (II) above, then they are isomorphic.

Proof. There are eighteen cases, and since they are all similar we will do only one. So suppose $\mathcal{A}^{\prime}$ and $\because h$ are countable models of $T$ and they both satisfy, say (I)(b) and (II)(f). Let $\left\{a_{k} \mid i<\omega\right\}$ and $\left\{b_{i} \mid i<\omega\right\}$ be enumerations of $\left|\hat{A}^{\prime}\right|$ and $\mid$ i $\hat{i} \mid$, respectively, such that $a_{0}, b_{0}$ are elements satisfying (I)(b) in $\left(f^{\prime}, b^{\prime}\right.$, respectively. By the usual kind of argument we build $f:\left(f^{\prime} \equiv\right.$ induction on $s$.

$$
f_{0}={ }_{\mathrm{df}}\left\langle a_{0}, b_{0}\right\rangle .
$$

Suppose $f_{i}$ for $i<2 n$ has been defined satisfying:

(1) $\cup_{i<2 n} f_{i}\left(a_{j_{r}}\right)=b_{k_{r}}, r<2 n$;

(2) $\left\{a_{0}, \ldots, a_{n-1}\right\} \subseteq\left\{a_{j_{1}} \mid i<2 n\right\}$;

(3) $\left\{b_{0}, \ldots, b_{n-1}\right\} \subseteq\left\{b_{k}, i<2 n\right\}$; and

(4) $\left\langle a_{j_{1}}, \ldots, a_{j_{2 n},}\right\rangle$ and $\left\langle b_{k_{1}}, \ldots, b_{k_{2 n} 1}\right\rangle$ realize the same type in $\left.4^{\prime}, \omega^{\prime}\right)$, respectively.

Let $\Gamma, \Sigma$ be the types realized in $t^{\prime}$ by $\left\langle a_{j_{1}}, \ldots, a_{j_{2 n},}\right\rangle$ and $\left\langle a_{j_{1}}, \ldots, a_{j_{2 n},}, a_{n}\right\rangle$, respectively. It is enough to show that there is a formula $\theta\left(x_{1}, \ldots, x_{2 n}\right) \in$ $\Sigma\left(x_{1}, \ldots, x_{2 n}\right)$ such that

$$
\Gamma\left(c_{1}, \ldots, c_{2 n-1}\right)+\theta\left(c_{1}, \ldots, c_{2 n-1}, x\right) \rightarrow \sigma\left(c_{1}, \ldots, c_{2 n-1}, x\right)
$$

for all $\sigma\left(x_{1}, \ldots, x_{2 n}\right) \in \Sigma\left(x_{1}, \ldots, x_{2 n}\right)$. For suppose we have done so; then obviously, $\exists x_{2 n} \theta\left(x_{1}, \ldots, x_{2 n}\right) \in \Gamma\left(x_{1}, \ldots, x_{2 n-1}\right)$, and therefore $\Re \vDash$ $\exists x \theta\left(b_{k_{1}}, \ldots, b_{k_{2 n-1}}, x\right)$. Then simply define $f_{2 n}={ }_{\mathrm{df}} f_{2 n-1} \cup\left\{\left\langle a_{n}, b\right\rangle\right\}$ for a $b \in|\Re|$ such that $\mathscr{B} \vDash \theta\left(b_{k_{1}}, \ldots, b_{k_{2 n-1}}, b\right)$.

There are several different cases for finding the desired $\theta$.

(A) $\Gamma_{g}^{X}\left(x_{2 n}\right) \subset \Sigma\left(x_{1}, \ldots, x_{2 n}\right)$ for some $X \neq L, g \neq f^{*}$.

It is easy to see by Lemma 2 that $\Gamma_{g}^{X}\left(x_{2 n}\right)$ is then a principal type and is thus generated by a single formula. Therefore by Lemmas 1 and $3, \Sigma\left(x_{1}, \ldots, x_{2 n}\right)$ is certainly uniquely determined by its containment of $\Gamma\left(x_{1}, \ldots, x_{2 n-1}\right)$, the generator for $\Gamma_{g}^{X}\left(x_{2 n}\right)$, and finitely many additional formulas. Thus $\theta\left(x_{1}, \ldots, x_{2 n}\right)$ can be taken as the conjunction of that generator and those finitely many additional formulas. 
(B) $\Gamma_{\eta}^{L}\left(x_{2 n}\right) \subset \Sigma\left(x_{1}, \ldots, x_{2 n}\right)$.

We first wish to eliminate the possibility that, in addition, $L_{\left\langle^{\eta}{ }^{\eta}\right.}\left(x_{2 n}, x_{1}\right) \in$ $\Sigma\left(x_{1}, \ldots, x_{2 n}\right)$. Assume otherwise in order to obtain a contradiction. By the definition of our original model it is easy to see that $\exists y R_{\langle\rangle}^{\eta}\left(x_{2 n}, y\right) \in \Gamma_{\eta}^{L}\left(x_{2 n}\right)$. So fix an $a^{\prime} \in|\mathfrak{Q}|$ satisfying $\mathscr{Q} \vDash R_{\zeta\rangle}^{\eta}\left(a_{n}, a^{\prime}\right)$. Then by property (XXXI),

$$
Q=L_{\zeta\rangle}^{\langle\rangle}\left(a^{\prime}, a_{0}\right) \text {. }
$$

But this together with properties (XV), (XII), and (XVII) contradict the choice of $a_{0}$. Thus, by property (XVIII),

$$
\mathbb{Q} F\left[L_{\eta}^{\langle\rangle}\left(a_{0}, a_{n}\right) \vee R_{\eta}^{\langle\rangle}\left(a_{0}, a_{n}\right)\right] .
$$

Then by properties (XXV), (XXVI), and (II)-(V),

$$
\mathcal{Q} \vDash \forall x\left[L_{\eta}^{\langle\rangle}\left(a_{0}, x\right) \vee R_{\eta}^{\langle\rangle}\left(a_{0}, x\right)\right] \rightarrow \neg S_{\eta\langle i\rangle} \text { for all } i<\omega .
$$

So again by Lemma $2\left[L_{\eta}^{\langle\rangle}\left(a_{0}, x_{2 n}\right) \vee R_{\eta}^{\langle\rangle}\left(a_{0}, x_{2 n}\right)\right]$ generates $\Gamma_{\eta}^{l} \cdot\left(x_{2 n}\right)$ in the theory of $\left\langle Q, a_{0}\right\rangle$. Now the argument is just as it was in case (A).

(C) Otherwise-thus $\Gamma_{f^{*}}\left(x_{2 n}\right) \subset \Sigma\left(x_{1}, \ldots, x_{2 n}\right)$.

Note first that by Lemma 3 and its proof that the 2-type projection of $\Sigma\left(x_{1}, \ldots, x_{2 n}\right)$ in the variables, say $\left\langle x_{1}, x_{2 n}\right\rangle$, is uniquely determined by the corresponding 1-type projections in case $\Gamma_{f^{*}}\left(x_{i}\right) \not \subset \Sigma\left(x_{1}, \ldots, x_{2 n}\right)$. Also, even if $\Gamma_{f^{*}}\left(x_{i}\right) \subset \Sigma\left(x_{1}, \ldots, x_{2 n}\right)$, Lemma 3 still shows that the 2-type projection is uniquely determined by the corresponding 1-type projections and its containment of one formula from $\left\{\left(x_{i}=\right.\right.$ $\left.\left.x_{2 n}\right),\left(x_{i}<x_{2 n}\right),\left(x_{2 n}<x_{i}\right)\right\}$. Thus in this case we simply abandon our search for $\theta\left(x_{1}, \ldots, x_{2 n}\right)$ and instead, using the fact that $<^{C_{B}}$ must be dense, find a $b \in \mid$ 级 such that:

(i) $b$ realizes $\Gamma_{f^{*}}$ in $\Re$;

(ii) if $\Gamma_{f^{*}}\left(x_{1}\right) \subset \Sigma\left(x_{1}, \ldots, x_{2 n}\right)$ and $\left(x_{i}<x_{2 n}\right) \in \Sigma\left(x_{1}, \ldots, x_{2 n}\right)$, then $9 B k b_{i}<b$;

(iii) as in (ii) except instead $\left(x_{2 n}<x_{i}\right) \in \Sigma\left(x_{1}, \ldots, x_{2 n}\right)$; then $9 \beta \vDash b<b_{i}$; and

(iv) as in (ii) except $\left(x_{i}=x_{2 n}\right) \in \Sigma\left(x_{1}, \ldots, x_{2 n}\right)$; then let $b=b_{i}$.

There can be no conflict here, since if, for instance, $b_{i}$ satisfies (ii) and $b_{j}$ satisfies (iii), then any $b \in \mid \Re$, such that $\mathscr{B} \vDash b_{i}<b \wedge b<b_{j}$, will automatically satisfy (i) by properties (XIX), (XII), (XIII), and (VI). Then $f_{2 n}={ }_{\mathrm{df}} f_{2 n-1} \cup\left\{\left(a_{n}, b\right)\right\}$. For $f_{2 n+1}$ we simply reverse the roles of $\mathcal{Q}$ and $\mathscr{B}$ in the above argument.

Thus $T$ has at most eighteen countable models up to isomorphism. In fact each of the combinations from (I) and (II) above are realizable, and so $T$ has exactly eighteen models. Let $\mathfrak{Q}_{x y}$ denote a countable model of $T$ satisfying (I) $(x)$ and (II) $(y)$. For $\mathfrak{Q}_{b y}$, let $b \in\left|\mathfrak{Q}_{b y}\right|$ be an element as in (I)(b); for $\mathbb{Q}_{x c}$ or $\mathbb{Q}_{x d}$ let $d$ be an element as in (II)(d); and for $\mathbb{Q}_{x c}$ or $\mathbb{Q}_{x e}$ let $e$ be an element as in (II)(e). Then from the above arguments it is routine to verify that each of the following structures is homogeneous:

(1) $\mathbb{Q}_{a a} ;$ (2) $\mathbb{Q}_{a b} ;(3)\left\langle\mathfrak{Q}_{a c}, d, e\right\rangle ;$ (4) $\left\langle\mathbb{Q}_{a d}, d\right\rangle ;$ (5) $\left\langle\mathscr{Q}_{a e}, e\right\rangle ;$ (6) $\mathbb{Q}_{a f} ;$

(7) $\left\langle Q_{b a}, b\right\rangle ;(8)\left\langle Q_{b b}, b\right\rangle ;(9)\left\langle Q_{b c}, b, d, e\right\rangle ;$ (10) $\left\langle Q_{b d}, b, d\right\rangle ;(11)\left\langle Q_{b e}, b, e\right\rangle$;

(12) $\left\langle\mathbb{Q}_{b f}, b\right\rangle ;$; (13) $\mathbb{Q}_{c a} ;(14) \mathbb{Q}_{c b} ;$ (15) $\left\langle\mathbb{Q}_{c c}, d, e\right\rangle ;(16)\left\langle\mathbb{Q}_{c d}, d\right\rangle$;

(17) $\left\langle\mathbb{Q}_{c e}, e\right\rangle ;(18) \mathbb{Q}_{c f}$. 
Also, by the above arguments, it is not difficult to show that $T$ is persistently finite. Finally, each of the types of $T$ is either recursive or Turing equivalent to $f^{*}$. With these observations and the results in Millar [3] each of the above structures is decidable exactly in either $N$ or $f^{*}$.

\section{REFERENCES}

1. C. C. Chang and H. J. Keisler, Model theory. American Elsevier, New York, 1973.

2. T. S. Millar, Foundations of recursive model theory, Ann. Math. Logic 13 (1978), 45-72.

3. . Type structure complexity and decidability, Trans. Amer. Math. Soc. 271 (1982), 73-81.

4. H. Rogers, Jr., Theory of recursive functions and effective computability. McGraw-Hill. New York, 1967.

Department of Mathematics, University of Wisconsin, Madison, Wisconsin 53706 